\title{
Novel Monopolar MFIE MoM-Discretization for the Scattering Analysis of Small Objects
}

\author{
Eduard Ubeda and Juan M. Rius, Member, IEEE
}

\begin{abstract}
We present a novel method of moments (MoM)-magnetic field integral equation (MFIE) discretization that performs closely to the MoM-EFIE in the electromagnetic analysis of moderately small objects. This new MoM-MFIE discretization makes use of a new set of basis functions that we name monopolar RaoWilton-Glisson (RWG) and are derived from the RWG basis functions. We show for a wide variety of small objects -curved and sharp-edged-that the new monopolar MoM-MFIE formulation outperforms the conventional MoM-MFIE with RWG basis functions.
\end{abstract}

Index Terms-Electromagnetic scattering, integral equations, method of moments (MoM), numerical analysis, radar cross sections (RCS).

\section{INTRODUCTION}

$\mathbf{T}$ HE method of moments (MoM) discretization of the electric field integral equation (EFIE) formulation with RaoWilton-Glisson (RWG) basis functions [1], a low order example of divergence-conforming set [2], [3], is widely used in the analysis of objects to obtain scattering parameters such as the radar cross section (RCS). However, it is well-known that there is a low-frequency breakdown problem with this operator because the condition number blows up at very low frequencies. In order to overcome such problem, a loop and tree basis decomposition [4] was introduced, allowing the solution of EFIE-problems at extremely low frequencies. Recently, Yunhua Zhang et al. [5] have presented an approach based on the loop-tree bases and the perturbation method for the MFIE formulation.

The use of MoM-EFIE with RWG basis functions is valid as long as the condition number of the system of equations can be managed, which depends on the discretization size adopted. In the scattering analysis of moderately small objects, the EFIE operator with RWG basis functions can be successfully used in practice for dimensions bigger than one hundredth of the wavelength [1].

Since the condition number of the MoM-operators derived from the MFIE is stable when the frequency decreases, one may also consider the use of a MoM-MFIE formulation in the analysis of such problems. However, it has been reported some clear disagreement of the conventional MoM-MFIE respect to the MoM-EFIE [6], [12] in the analysis of moderately small objects

Manuscript received September 23, 2004; revised September 29, 2005. This work was supported by the "Departament d'Universitats, Recerca i Societat de la Informació (DURSI)" under "Distinció de la Generalitat de Catalunya per a la Promoció de la Recerca Universitaria" and the Spanish "Comisión Interministerial de Ciencia y Tecnología (CICYT)" through the "Ramón y Cajal" Programme and the grants TIC 2001-2364-C01-01, TIC 2003-09317-C03-03.

The authors are with the Signal Theory and Communications Department, Universitat Politécnica de Catalunya, 08034 Barcelona, Spain (e-mail: ubeda@tsc.upc.edu).

Digital Object Identifier 10.1109/TAP.2005.861529 in scattering problems. This discrepancy, for which a heuristical correction is provided in [6], becomes especially evident in the analysis of moderately small sharp-edged objects. The conventional MoM-MFIE must introduce some error at sharp edges because as the dimensions of the object decrease, the relative influence of the sharp edges in the overall result is more important, and the discrepancy with MoM-EFIE becomes more noticeable [6], [9].

Recently, a MoM-MFIE formulation with a low-order curlconforming set of basis functions and very accurate Kernelintegration [8], [9] has shown to be better performing than the conventional MoM-MFIE with RWG basis functions in the analysis of electrically small sharp-edged objects. In this paper we present a novel MFIE formulation based on a new set of basis functions-monopolar RWG-derived from the conventional formulation with RWG basis functions, with very similar performance to the EFIE operator for any type of small object, provided that the electrical size is not too small to keep the EFIE condition number bounded.

\section{INTEGRAL OPERATORS: EFIE AND MFIE}

The formulations for the EFIE and for the MFIE are derived from the electric and magnetic field boundary conditions over the surface of the scatterer

$$
\begin{aligned}
\text { MFIE }: \vec{J} & =\hat{n} \times \vec{H}=\hat{n} \times \vec{H}^{i}+\hat{n} \times \vec{H}^{s} \\
\text { EFIE }: 0 & =[\vec{E}]_{\tan }=\left[\vec{E}^{i}+\vec{E}^{s}\right]_{\tan }
\end{aligned}
$$

where $\hat{n}$ denotes the normal vector to the surface of the scatterer and $\vec{E}^{i}, \vec{H}^{i}$ and $\vec{E}^{s}, \vec{H}^{s}$ stand for the incident and the scattered fields, respectively. By [ $]_{\tan }$ we mean the field component tangential to the surface.

The Galerkin approach in MoM requires the testing of the fields and the expansion of the current with the same set of basis functions $\left\{\vec{f}_{n}\right\}$. The impedance elements $z_{m n}$ become then for each operator

$$
\begin{aligned}
\text { MoM-MFIE : } z_{m n}= & \frac{\left\langle\vec{f}_{m}, \vec{f}_{n}\right\rangle}{2} \\
& -\left\langle\vec{f}_{m}, \hat{n}_{m} \times \int_{T_{n}^{+} \cup T_{n}^{-}} \overrightarrow{f_{n}} \times \nabla^{\prime} G d s^{\prime}\right\rangle \\
\text { MoM-EFIE : } z_{m n}= & -j k \eta\left\langle\vec{f}_{m}, \int_{T_{n}^{+} \cup T_{n}^{-}} G \vec{J} d s^{\prime}\right\rangle \\
& -j \frac{\eta}{k}\left\langle\vec{f}_{m}, \int_{T_{n}^{+} \cup T_{n}^{-}} \nabla G \nabla^{\prime} \cdot \vec{f}_{n} d s^{\prime}\right\rangle
\end{aligned}
$$


where the general expression for the inner-product stands for

$$
\left\langle\vec{f}_{m}, \vec{S}\right\rangle=\int_{T_{m}^{+} \cup T_{m}^{-}} \vec{f}_{m} \cdot \vec{S} d s
$$

and $\int_{T_{n}^{+} \cup T_{n}^{+}}, \int_{T_{m}^{+} \cup T_{m}^{+}}$denote the integration over the $n$th and $m$ th source- and field-domains, which embrace a pair of triangles sharing an edge, $T^{+}$and $T^{-}$[1].

From the MoM-expressions above we can readily build several MoM-integral operators in accordance with the set of basis functions adopted. The RWG basis functions, a low-order example of divergence-conforming set, leads to the EFIE and MFIE[RWG] operators [1], [10]. The use in the MoM-MFIE formulation of a low-order curl-conforming set like the one derived from RWG by cross-multiplying by the normal unit vector to the surface ( $n x \mathrm{RWG}$ ) results in the operator MFIE[ $n x \mathrm{RWG]}$ [7]-[9].

In order to establish a fair comparison between the performance of the different operators it is critical to ensure the accurate computation of the impedance elements. This is particularly important in our case of interest of electrically small objects. From the decomposition of the kernels of EFIE and MFIE

$$
\begin{aligned}
\text { MFIE }: \nabla G= & -(4 \pi)^{-1}\left(\vec{r}-\vec{r}^{\prime}\right) \\
& \times\left(R^{-3}+0.5 k^{2} R^{-1}-0.5 j k^{3}+O(R)\right)
\end{aligned}
$$

$$
\text { EFIE : } G=(4 \pi)^{-1}\left(R^{-1}-j k+O(R)\right)
$$

we see that the high-order terms $R^{-3}$ or $R^{-1}$ are singular when $m=n$ and almost singular for pairs of basis and testing functions involving very close triangles. In the MFIE formulation, the integration of the almost singular contributions coming from very near interactions between basis functions on noncoplanar triangles must be carried out in a very accurate manner [9].

\section{NOVEL MONOPOLAR MFIE OPERATOR}

In all the operators of the previous section the impedance elements result from the interactions between pairs of triangles sharing an edge. This is due to the usual definition of the operators because it is a consequence of the definition of the sets of basis functions, which force either the normal component or the tangential component of the current across the edge to be constant [2], [3]. Therefore, the number of edges is equal to the number of unknowns.

In this paper, we propose a different strategy to establish the set of basis functions and build the MoM-MFIE formulation. The new set of basis functions adopts the definition for the RWG basis functions inside each triangle [1] but no constraint about the continuity of the normal component of the current across the edge is imposed (see Fig. 1). Therefore, we set independent basis functions, and thus different unknowns, at each side of the edge.

We can intuitively see these new basis functions as elementary electric monopoles at both sides of the edges. Since they are derived from the RWG set, we name them as monopolar RWG (see Fig. 1). In contrast, the RWG basis functions can be seen as elementary dipoles assigned to each edge because by definition the charge accumulation across the edge is prevented and because total null charge over the two triangles is ensured.
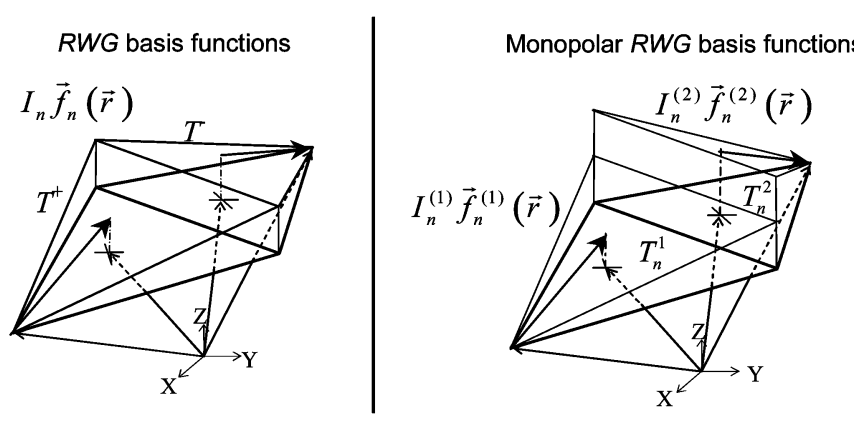

Fig. 1. Definition of the monopolar RWG basis functions.

The new MoM-MFIE formulation proposed in this paper comes from setting the monopolar RWG set as testing and basis functions in a Galerking approach in MoM. We thus name the new formulation as monopolar MoM-MFIE (MFIE[mono]). Note that it has to compute each of the four triangle-to-triangle interactions $\left(T_{m}^{1}-T_{n}^{1}, T_{m}^{1}-T_{n}^{2}, T_{m}^{2}-T_{n}^{1}, T_{m}^{2}-T_{n}^{2}\right)$ that form one edge-to-edge interaction $(m-n)$ in the conventional MFIE[RWG] operator (see Fig. 1). Therefore the definition of the impedance elements for the new operator is closely linked to the definition in (3) and becomes

$$
\begin{aligned}
& \text { monopolar MFIE }: z_{m^{i} n^{j}} \\
& \quad=\frac{\left\langle\vec{f}_{m}^{i}, \vec{f}_{n}^{j}\right\rangle}{2}-\left\langle\vec{f}_{m}^{i}, \hat{n}_{m}^{j} \times \int_{T_{n}^{i}} \vec{f}_{n}^{i} \times \nabla^{\prime} G d s^{\prime}\right\rangle \\
& i=1,2 j=1,2
\end{aligned}
$$

where the surface source-integrals $\int_{T_{n}^{1}}$ or $\int_{T_{n}^{2}}$ and the testing integrals $\int_{T_{m}^{1}}$ or $\int_{T_{m}^{2}}$ of the inner-product now embrace one of the two triangles ( $T^{1}$ or $T^{2}$ ) defining either the $m$-edge or the $n$-edge in the MFIE[RWG] operator.

For a given meshing, the monopolar MoM-MFIE formulation, when compared with the dipolar MFIE[RWG], doubles the number of unknowns and multiplies the memory storage for $Z$ by four. Indeed, while we set $I_{n}$ as the normal component across the edge in the conventional MFIE[RWG], in MFIE[mono] we set two different unknowns $I_{n}^{(1)}$ and $I_{n}^{(2)}$ at both sides of the edge $n$ corresponding to the normal component of the current at both sides of the edge $n$ (see Fig. 1). Similarly, the impedance element $z_{m n}$ of MFIE[RWG] leads to four elements $z_{m^{1} n^{1}}, z_{m^{1} n^{2}}, z_{m^{2} n^{1}}, z_{m^{2} n^{2}}$ in MFIE[mono]. This increase of the number of unknowns is not a major problem in general in the analysis of electrically small objects because the number of unknowns is normally moderate and because the matrix fill-time is longer than the linear system solution time. The time for generating the matrix $Z$ is moreover analogous for MFIE[mono] and for MFIE[RWG] because, since the impedance elements in MFIE[RWG] come from the combination of four impedance elements of the new operator, we end up managing the same computational load. Additionally, it must be remarked that for a given discretization, the RCS-performance with the new monopolar MoM-MFIE formulation is closer to EFIE than making the meshing twice finer and solve the problem with the conventional MFIE[RWG], which equivalently doubles the number of unknowns as well. 
In the analysis of sharp-edged objects, we can define a hybrid formulation by setting monopolar basis functions only for those edges between noncoplanar triangles. These are the edges that affect most in the discrepancy of MFIE[RWG] respect to EFIE [6], [9]. We then obtain a hybrid MoM-MFIE formulation that shows an RCS-performance very similar to the pure monopolar formulation and provides less computational load because the number of unknowns is reduced drastically. We name this formulation as MFIE[hybrid].

\section{Results}

To prove the validity of the monopolar MoM-MFIE formulation we show results for a significant variety of electrically small curved and sharp-edged objects (a sphere, a cube, a prism and a cone). Since the objects are not too small to make the EFIE condition number blow up, EFIE is adopted as the reference result. For the case of noncurved sharp-edged objects we also show the performance of MFIE[ $n x$ RWG] with very accurate Kernel-integration, which shows an RCS-performance very close to EFIE [9]. The impinging plane wave is $x$-polarized and with $+z$-propagation and $\lambda=1 \mathrm{~m}$. To establish a fair and thorough comparison between the different formulations, we present for all the objects a far-field and a near-field testing.

The far-field testing stands for the comparison of the RCS patterns due to the different formulations. As it is well-known, there may be cancellation of errors of the current in the farfield computation that may lead to conceal the real behavior of each formulation. Therefore, as suggested in [13], we need to ensure most accuracy in the computation of the current to yield a reliable far-field computation. We thus minimize these error sources through the direct noniterative inversion of the $Z$ matrix, through the adoption of fine and nondistorted meshing grids, through the accurate computation of the impedance elements and through the selection of testing bodies with polyhedrical shape to allow a proper modeling with flat-facet meshes.

Special attention must be cast into the accurate computation of the impedance elements. For the RWG-based formulations EFIE[RWG], MFIE[RWG] and MFIE[mono] we carry out the analytical integration of the high-order terms in (6) and (7) for any pair of field-source interactions as described in [1], [10], [11]. Similarly, the computation of MFIE $[n x \mathrm{RWG}]$ is also undertaken with an accurate Kernel-integration technique described in [9]. We have computed the inner integral of the remaining low-order terms in (6) and (7) and the outer integral with the same number of points through accurate Gaussian quadrature rules [15]. We have set the number of integrating points by choosing the minimum order of the quadrature rule for which the RCS-performance barely varies. We have then observed that MFIE[RWG] and MFIE[mono] need more integrating points to reach a stable result than EFIE and $\mathrm{MFIE}[n x \mathrm{RWG}]$. In particular, we have adopted six points for the analysis of all the testing objects with EFIE and MFIE[ $n x$ RWG] and for the analysis of the sphere, the prism and the cube with MFIE[RWG] and MFIE[mono]. For the analysis of the cone with MFIE[RWG] and MFIE[mono] nine points have been required.

We carry out the near-field testing by computing the relative error in accomplishing the equivalence principle inside the selected objects. Since the electromagnetic fields inside the body must be null, we have computed the total electric field (for the EFIE formulation) and the total magnetic field (for the MFIE formulations) at a set of testing points inside: $\left\{\vec{r}_{\text {test }}^{n}\right\}$. We choose this set of points for each object to be regularly spread so that they fill up the whole volume of the object; we then compute the quantity

$$
\text { err_in }=\frac{\left[\sum_{n=1}^{\text {Ntest }}\left|\vec{F}^{i}\left(\vec{r}_{\text {test }}^{n}\right)+\vec{F}^{S}\left(\vec{r}_{\text {test }}^{n}\right)\right|^{2}\right]^{1 / 2}}{\left[\sum_{n=1}^{\text {Ntest }}\left|\vec{F}^{i}\left(\vec{r}_{\text {test }}^{n}\right)\right|^{2}\right]^{1 / 2}}
$$

where $\vec{F}^{i}$ and $\vec{F}^{S}$ stand for the electric or magnetic incident and scattered fields and Ntest denotes the number of testing points employed. Therefore, err_in represents an independent measure for the accuracy of each formulation that complements the far-field observations in the RCS patterns. Moreover, we can assess if EFIE is valid and still unaffected by the low-frequency breakdown. Again, in the computation of the scattered magnetic and electric fields in (9) we must provide high accuracy in order to obtain reliable evidences about the behavior of the formulations. The scattered magnetic field due to the dipolar RWG and monopolar RWG sets is computed accurately through the analytical source-integral of the highest-order terms of the Kernel together alongside a six-point quadrature rule for the remaining low-order terms [10]. However, the accurate procedure used in the computation of the impedance elements in EFIE and MFIE[ $n x$ RWG] cannot be now applied in (9). Indeed, since the scattered fields are not tested with the outer integral, we cannot take advantage of swapping the source- and field-integrals to make the singularity extraction easier [1], [9]. Therefore, we have computed the electric scattered field due to RWG by integrating analytically the highest-order terms in $\nabla G$ [10] together with a six-point quadrature rule for the remaining terms. Similarly, we have computed numerically the magnetic scattered field due to $n x$ RWG with a 61-point quadrature rule [15]. This integrating criterion is not suitable for testing the magnetic field on the surface of a sharp-edged object [9], but, since in these near-field computations we need to obtain the field inside the volume, this rule turns out sufficiently accurate for the set of testing points adopted.

\section{A. Curved Objects}

We show the RCS for an electrically small sphere with radius $0.2 \lambda$ and meshed with 128 triangles. It is analyzed with the operators EFIE, MFIE[RWG] and MFIE[mono]. In view of Figs. 2 and 3, the agreement between EFIE and MFIE[mono] is very good. The discrepancy of the conventional MFIE[RWG] appears clearly because the moderately small dimensions of the sphere allow a somewhat inaccurate modeling of the curvature that introduces edges with significant degree of sharpness. We have observed that for electrically bigger spheres, with smaller triangles and better modeling of the curvature, the degree of sharpness of the edges introduced in the discretization diminishes and so does the RCS-discrepancy in MFIE[RWG].

The near-field testing of EFIE, MFIE[RWG] and MFIE[mono] for the sphere is carried out in a grid of 198 testing points regularly spread over three concentric spherical surfaces of radii $0.05,0.1$, and $0.15 \mathrm{~m}$ placed inside the testing 


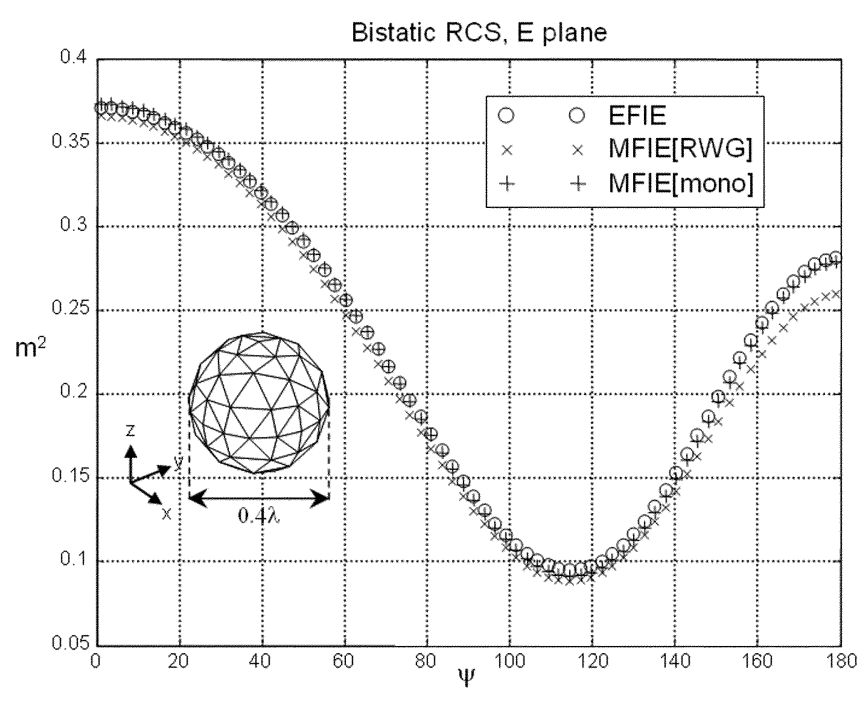

Fig. 2. Bistatic E plane RCS for a sphere with radius $0.2 \lambda$ meshed with 128 triangles.

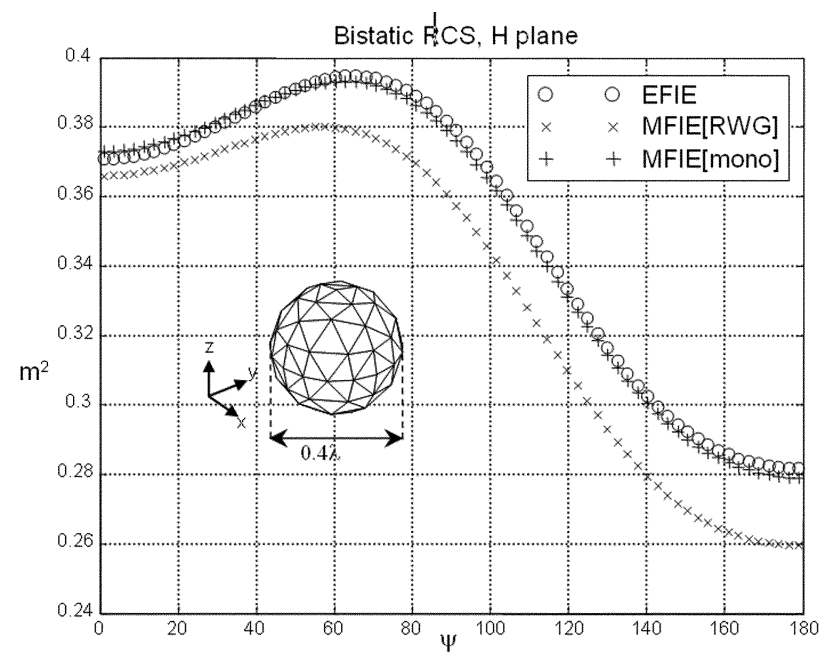

Fig. 3. Bistatic $\mathrm{H}$ plane RCS for a sphere with radius $0.2 \lambda$ meshed with 128 triangles.

object (see Fig. 4). In Fig. 5, we show that MFIE[mono] shows a smaller err_in than MFIE[RWG] for both the coarse discretization with 128 tiangles and the fine discetization of 512 triangles. Interestingly, the values of err_in for MFIE[mono] and MFIE[RWG] become much more similar for the fine discretization. In this case, with better modeling of the curvature, the sharp-edges arising in the discretization become milder and therefore MFIE[RWG] behaves much better.

\section{B. Noncurved Sharp-Edged Objects}

The analysis of noncurved sharp-edged objects is convenient in order to make a fair comparison of the behavior of the different operators with planar basis functions: the sets RWG, $n x$ RWG and the monopolar RWG. All these basis functions are defined over flat-facet meshes such as triangles and they are therefore suitable to model geometries with polyhedrical shape. Since cubes and prisms do not allow perfectly regular meshings with

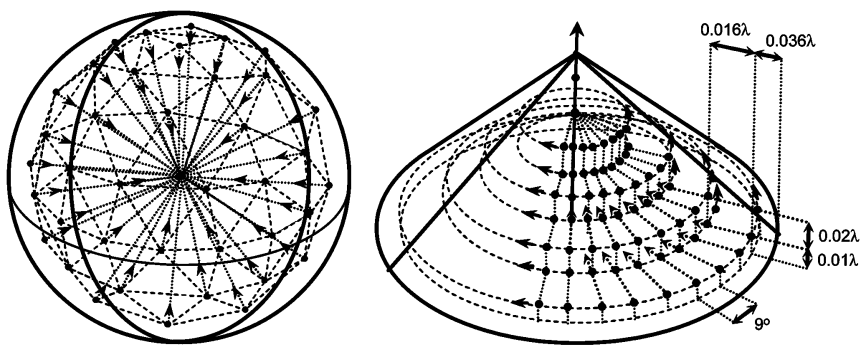

Fig. 4. Distribution of testing points inside the volume of the sphere and the cone.

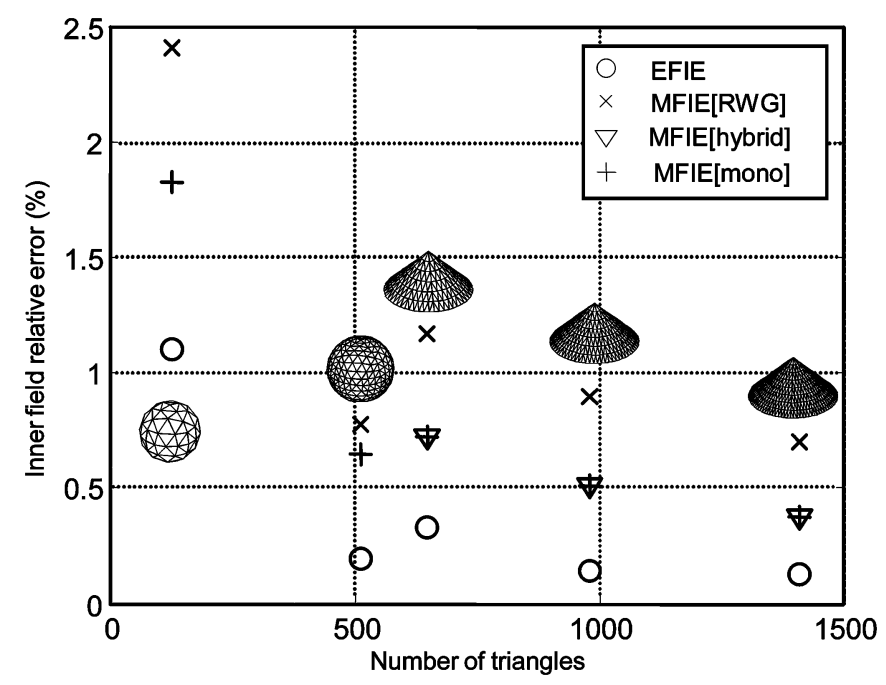

Fig. 5. Relative error ( $\mathrm{err}$ in) inside the sphere with radius of $0.2 \lambda$ and the cone with radius and height $0.1 \lambda$ for several discretizations.

equilateral triangles, we have chosen fairly nondistorted discretizations with very similar lengths of the sides, with two sides of the triangles with equal size in most of the cases. Thanks to this rigorous modeling of the geometry, we can focus only on the way the different formulations expand the current and generate the fields. For this type of objects, MFIE[ $n x$ RWG] show very similar RCS-performance with respect to EFIE, overcoming the known discrepancy arising in MFIE[RWG] [9]. In Figs. 6 and 7, we show the RCS for a moderately small prism with dimensions $0.1 \lambda \times 0.1 \lambda \times 0.05 \lambda$ meshed with 336 triangles resulting from the numerical analysis with EFIE, MFIE[RWG], MFIE[ $n x$ RWG] and MFIE[mono]. The performance of the reference operators EFIE and MFIE $[n x \mathrm{RWG}]$ is much more similar to MFIE[mono] than to the conventional MFIE[RWG]. In Figs. 8 and 9, we show the RCS for an electrically bigger cube with side $0.5 \lambda$ and the discrepancy in MFIE[RWG] is still very clear. Again, MFIE[mono] performs very closely to EFIE and MFIE[ $n x$ RWG]. Even if the improvement of the RCS-performance is very evident, it must be pointed out that MFIE[mono] doubles the number of unknowns respect to MFIE[RWG] with the same discretization. Let us look into this issue more carefully. If we assess the RCS-performance of MFIE[hybrid] for the prism and the cube (Figs. 6-9), we see that MFIE[hybrid] is still better performing than MFIE[RWG] and with very little deviation respect to MFIE[mono]. In view of this fact we realize again the important influence of the sharp-edges on the discrepancy of the conventional MFIE[RWG] with respect to EFIE. 


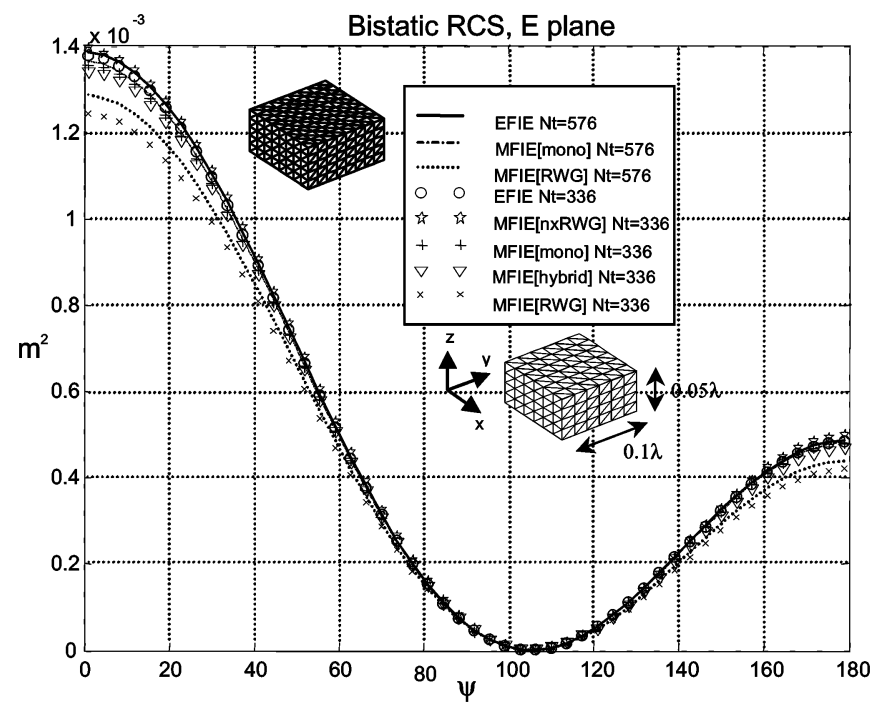

Fig. 6. Bistatic E plane RCS for a prism with dimensions $0.1 \lambda \times 0.1 \lambda \times 0.05 \lambda$ meshed with 336 and 576 triangles.

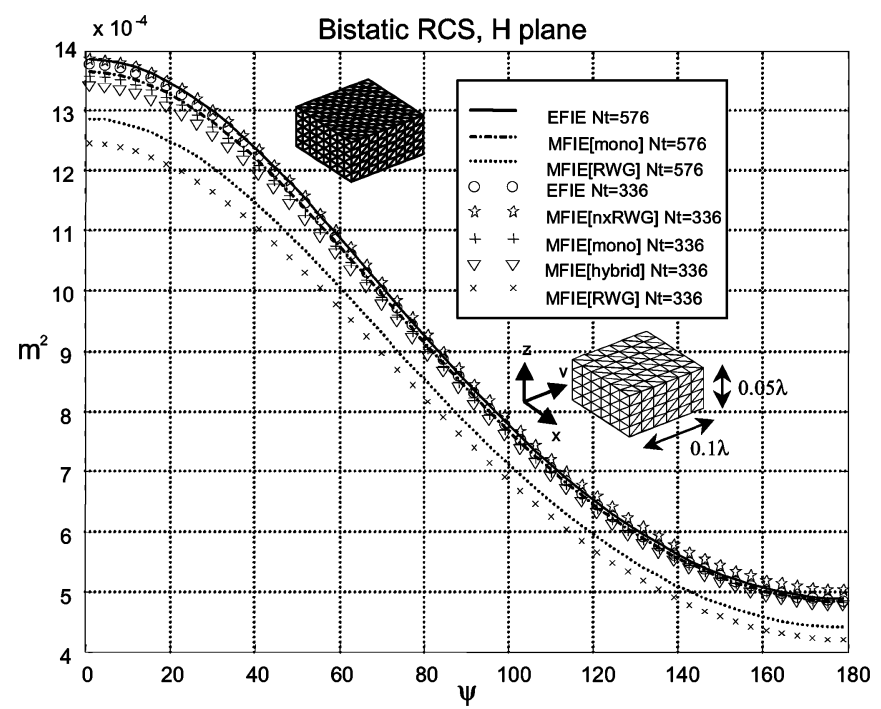

Fig. 7. Bistatic $\mathrm{H}$ plane RCS for a prism with dimensions $0.1 \lambda \times 0.1 \lambda \times 0.05 \lambda$ meshed with 336 and 576 triangles.

MFIE[hybrid] excels as a very effective formulation because for the prism it defines 568 unknowns (MFIE[RWG] makes use of 504) and for the cube with side $0.5 \lambda$ it requires 510 unknowns (MFIE[RWG] makes use of 450), many less than MFIE[mono] (1008 and 900 unknowns, respectively) and with fairly similar performance. Furthermore, we want to emphasize that the conventional MFIE[RWG] formulation defining less unknowns than MFIE[mono] shows no real advantage in terms of reliable RCS-performance. If we set a finer meshing for the prism of dimensions $0.1 \lambda \times 0.1 \lambda \times 0.05 \lambda$ by defining 576 triangles, the RCS-performance of the conventional MFIE[RWG] operator is still more distant to EFIE than MFIE[mono] for the previous coarser discretization with 336 triangles (see Figs. 6 and 7). Similarly, if we set a finer meshing of 768 triangles for the cube with side $0.5 \lambda$ (see Figs. 8 and 9),MFIE[RWG] cannot catch up with MFIE[mono] and the coarser meshing of 300 triangles either. Finally, in view of Figs. 6 and 7, it is interesting to remark that MFIE[mono] and a meshing finer for the prism performs in a very similar way as with the coarser discretization just like

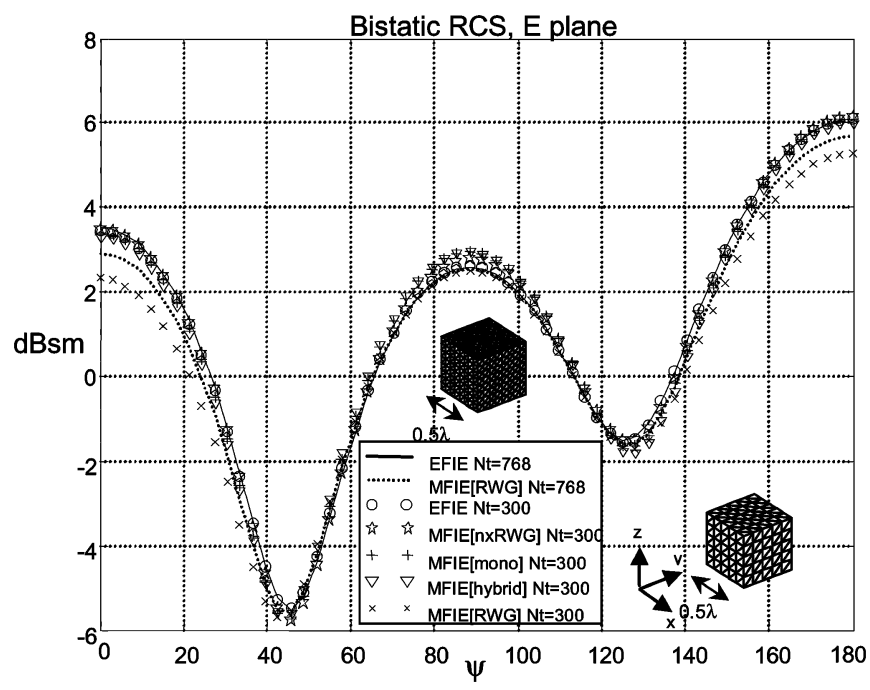

Fig. 8. Bistatic E plane RCS for a cube with side $0.5 \lambda$ meshed with 300 and 768 triangles.

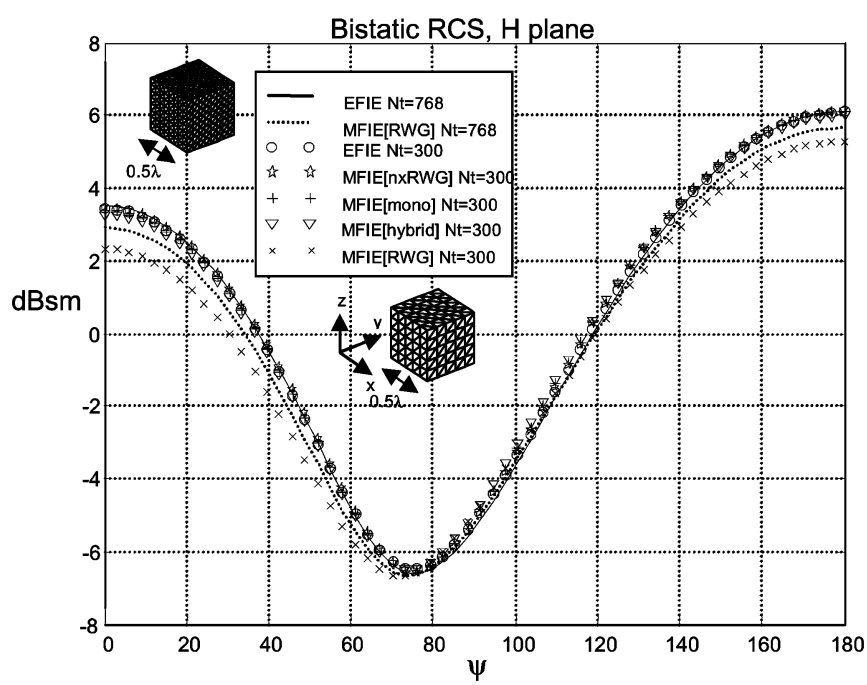

Fig. 9. Bistatic $\mathrm{H}$ plane RCS for a cube with side $0.5 \lambda$ meshed with 300 and 768 triangles.

the EFIE operator, which is again a proof of its robustness and validity. MFIE[RWG], in contrast, shows a remarkable variation of RCS-performance as we make the meshing finer.

The near-field testing of EFIE, MFIE[ $n x$ RWG], MFIE[RWG], MFIE[hybrid] and MFIE[mono] for the prism and the cube is carried out in grids of 405 and 216 testing points, respectively, regularly spread over the volume inside the testing objects (see Fig. 10). The spatial periodicities in the grids adopted for the cube and the prism are $D_{x}=$ $D_{y}=D_{z}=0.0714 \lambda$ and $D_{x}=D_{y}=D_{z}=0.01 \lambda$, respectively. We show in Fig. 11 for the prism and in Fig. 12 for the cube the relative inner field error for different discretizations. As expected, EFIE and MFIE[ $n x$ RWG] show the minimum error, which corroborates their adoption as references and excludes the low-frequency failure for EFIE. Similarly, in accordance with the far-field evidences, MFIE[RWG] offers the poorest figures for all the discretizations and MFIE[mono] outperforms them drastically. Note that since the management of the computation of the integrals arising in MFIE[RWG] and MFIE[mono] is identical, the remarkable improvement on the 


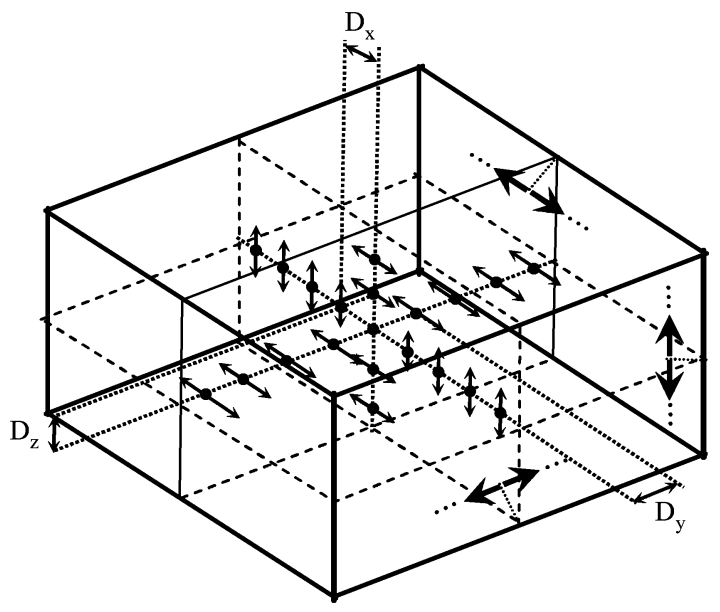

Fig. 10. Distribution of testing points inside the volume of the cube or the prism.

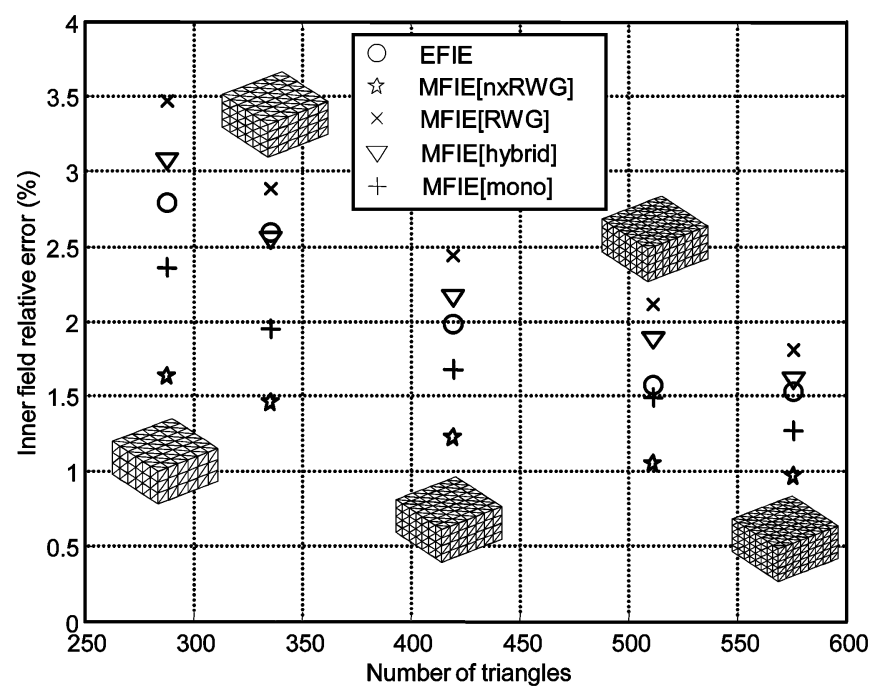

Fig. 11. Relative error (err in) inside the prism with dimensions $0.1 \lambda \times$ $0.1 \lambda \times 0.05 \lambda$ for several discretizations.

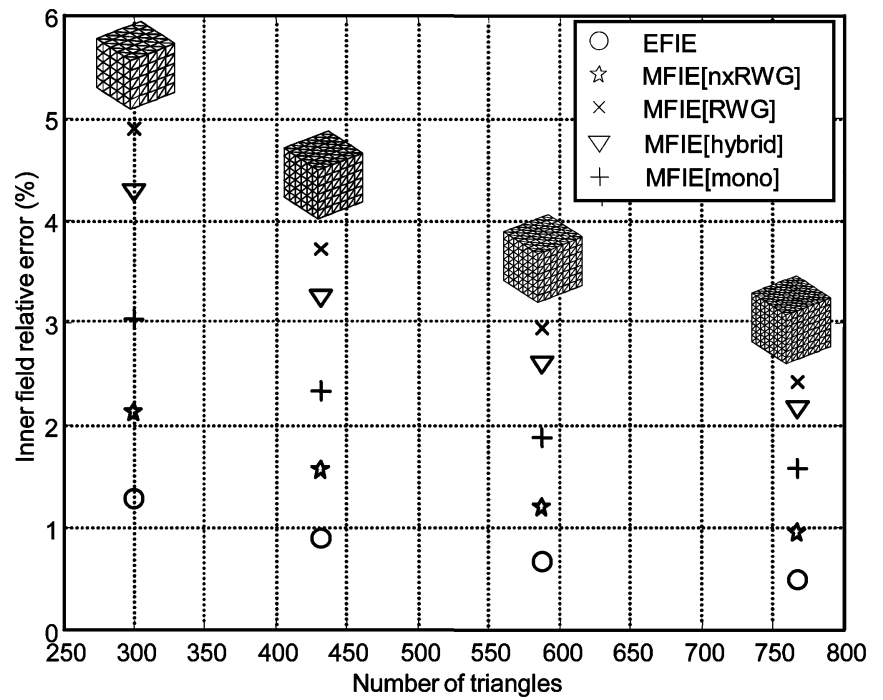

Fig. 12. Relative error (err_in) inside the cube with side $0.5 \lambda$ for several discretizations.

inner-field error values with MFIE[mono] shows the better suitability of the monopolar choice.

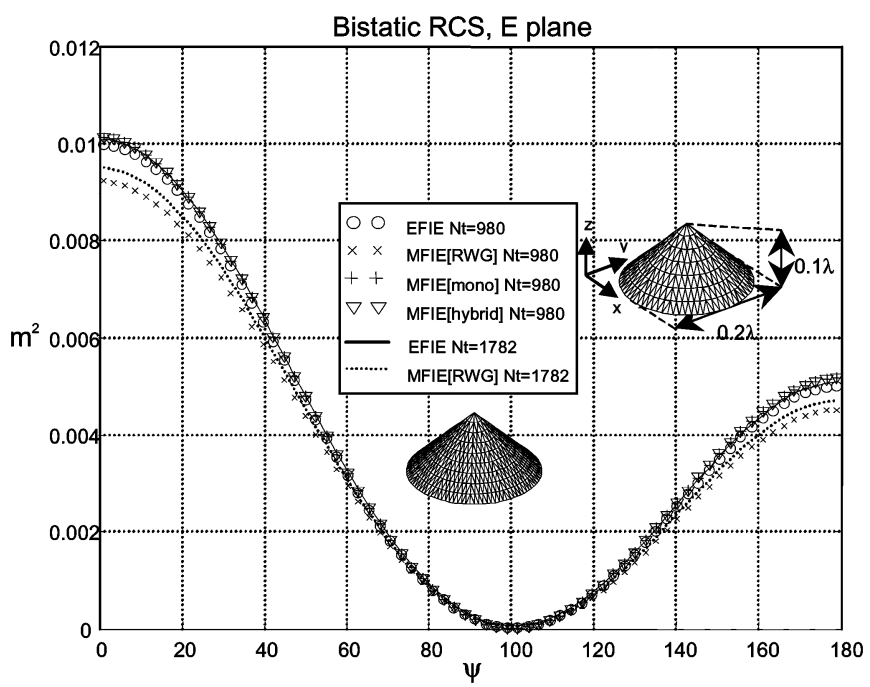

Fig. 13. Bistatic E plane RCS for a cone with height $0.1 \lambda$ and basis radius $0.1 \lambda$ meshed with 980 and 1782 triangles.

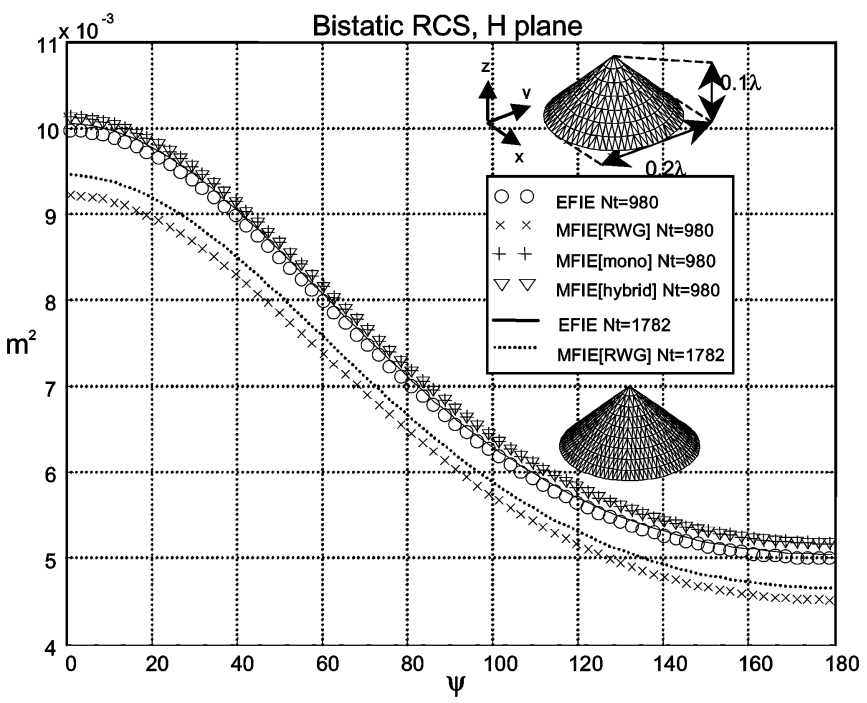

Fig. 14. Bistatic $\mathrm{H}$ plane RCS for a cone with height $0.1 \lambda$ and basis radius $0.1 \lambda$ meshed with 980 and 1782 triangles.

\section{Partially Curved Sharp-Edged Objects}

We show the performance of MFIE[mono] compared to EFIE and MFIE[RWG] for the case of a cone with diameter $0.2 \lambda$ and height $0.1 \lambda$. In Figs. 13 and 14, we show the RCS for two different discretizations of 980 and 1782 triangles. We choose these very fine discretizations to exclude the inappropriate modeling of the curvature of the cone in the differences of performance from the different formulations. We corroborate the observations presented for the previous cases: 1) MFIE[mono] follows the RCS pattern very closely to EFIE in comparison with the conventional MFIE[RWG]; 2) MFIE[hybrid], with monopolar RWG basis functions only at sharp-edges, shows very good RCS-agreement with the MFIE[mono], with monopolar basis functions applied to all the edges; 3) the RCS-performance of MFIE[RWG] and finer discretization is clearly insufficient to overcome the RCS-performance of MFIE[mono] operator and coarser discretization. 
The near-field testing of EFIE, MFIE[ $n x$ RWG], MFIE[RWG], MFIE[hybrid] and MFIE[mono] for the cone is carried out in a grid of 205 testing points regularly distributed inside the volume of the cone (see Fig. 4). In Fig. 5, we see again that MFIE[mono] and MFIE[hybrid] outperform MFIE[RWG] and EFIE excels as the best performing formulation. It is interesting to remark the identical inner field performance of MFIE[mono] and MFIE[hybrid] for the cone.

The evidences obtained through these near-field and far-field observations support each other for all the objects tested and lead to the fact that the a priori imposition of normal continuity for the current across the edges is not really required when discretizing the MFIE formulation. The development of a monopolar RWG MoM-EFIE formulation is a challenging task because it must account for the presence of line charges in the edges of the discretization. However, our attempts to build a successful monopolar RWG MoM-EFIE formulation have turned out so far fruitless. Indeed, successful and widespread MoM-EFIE implementations [1], [16], [17] adopt a MoM-discretization imposing normal continuity of the current through the edges and thus free of line charges at subdomain boundaries.

Some authors like Carr et al. [14] have presented in the context of the EFIE formulation the half-RWG basis functions, which are only applicable to the very particular case of junctions. Therefore, they appear as the extension of the RWG basis functions to junctions, for which RWG were not originally conceived. Moreover, the half-RWG basis function in [14] must comply with the Kirchoff's current continuity requirements at the edge in the junction, in the same way as RWG do at the remaining edges of the discretization. Interestingly, in contrast, our monopolar RWG basis functions set independents unknowns at both sides for all the edges arising in the MoM-discretization of the MFIE formulation.

\section{CONCLUSION}

We present a novel MoM-MFIE discretization scheme that outperforms the conventional MFIE[RWG] formulation in the analysis of moderately small objects. We name this formulation monopolar MoM-MFIE because it is based on a new set of basis functions, the monopolar RWG. This set makes use of the RWG definition inside the triangles but it does not impose continuity of the normal component of the current across the edge: two different unknowns are defined for the normal component of the current at each side of the edge. Since the same computational effort must be applied to compute the impedance elements in both formulations, the time to generate the impedance matrix is similar. We show for different types of moderately small objects and with very accurate Kernel-integration that the RCS computed with this new monopolar MoM-MFIE formulation follow more closely those due to the EFIE, which can be taken as reference because the electrical dimensions of these objects are not small to make the condition number blow up. To complement these observations, we remark that the monopolar RWG MoM-MFIE formulation yields a magnetic field inside of these testing objects closer to zero than the conventional dipolar RWG MoM-MFIE formulation.
Even though the number of unknowns with respect to the conventional MFIE[RWG] formulation is doubled, we show with a few varied examples of sharp-edged objects that the RCS-performance of the new monopolar MoM-MFIE formulation under a given discretization follows more closely the RCS-performance of EFIE than the conventional MFIE[RWG] and a meshing twice finer. Furthermore, as we increase the degree of meshing of the objects, the RCS-results due to the monopolar MoM-MFIE formulation are stable, like the EFIE operator, while MFIE[RWG] struggles to catch up with them.

In the analysis of sharp-edged objects, we have also presented a MoM-MFIE formulation with the definition of monopolar RWG basis functions only at the sharp-edges of the meshing. This hybrid formulation shows an RCS-performance very similar to the RCS-performance of the MoM-MFIE formulation with monopolar basis functions defined at all the edges. This is an excellent property because it involves managing many less unknowns than the pure monopolar MoM-MFIE formulation with little deviation of the performance.

\section{REFERENCES}

[1] S. M. Rao, D. R. Wilton, and A. W. Glisson, "Electromagnetic scattering by surfaces of arbitrary shape," IEEE Trans. Antennas Propag., vol. AP-30, no. 3, pp. 409-418, May 1982.

[2] J. C. Nedelec, "Mixed finite elements in $R^{3}$," Numer. Mathem., vol. 35, pp. 315-341, 1980.

[3] R. D. Graglia, D. R. Wilton, and A. F. Peterson, "Higher order interpolatory vector bases for computational electromagnetics," IEEE Trans. Antennas Propag., vol. 45, no. 3, pp. 329-342, Mar. 1997.

[4] D. R. Wilton and A. W. Glisson, "On improving the electric field integral equation at low frequencies," in URSI Radioscience Meeting Dig., Los Angeles, CA, 1981, pp. 24-24.

[5] Y. Zhang, T. J. Cui, W. C. Chew, and J.-S. Zhao, "Magnetic field integral equation at very low frequencies," IEEE Trans. Antennas Propag., vol. 51, no. 8, Aug. 2003.

[6] J. M. Rius, E. Ubeda, and J. Parrón, "On the testing of the magnetic field integral equation with rwg basis functions in method of moments," IEEE Trans. Antennas Propag., vol. 49, no. 11, pp. 1550-1553, Nov. 2001.

[7] A. F. Peterson and L. C. Kempel, "Solution of the MFIE using curlconforming basis functions," in Proc. IEEE Antennas Propag. Symp., Jun. 2002.

[8] E. Ubeda and J. M. Rius, "Curl-conforming MFIE in the analysis of perfectly conducting sharply-edged objects," presented at the Proc. IEEE Antennas Propag. Symp., Jun. 2004.

[9] — , "MFIE MoM-formulation with curl-conforming basis functions and accurate Kernel-integration in the analysis of perfectly conducting sharp-edged objects," Microw. Opt. Technol. Lett., vol. 44, no. 4, Feb. 2005.

[10] R. E. Hodges and Y. Rahmat-Samii, "The evaluation of MFIE integrals with the use of vector triangle basis functions," Microw. Opt. Technol. Lett., vol. 14, no. 1, pp. 9-14, Jan. 1997.

[11] D. R. Wilton, S. M. Rao, A. W. Glisson, D. H. Schaubert, O. M. Al-Bundak, and C. M. Butler, "Potential integrals for uniform and linear source distributions on polygonal and polyhedral domains," IEEE Trans. Antennas Propag., vol. AP-32, no. 3, pp. 276-281, Mar. 1984.

[12] O. Ergul and L. Gurel, "Investigation of the inaccuracy of the MFIE discretized with the RWG basis functions," presented at the Proc. IEEE Antennas Propag. Symp., Jun. 2004.

[13] C. P. Davies and K. F. Warnick, "Error analysis of 2-D MoM for MFIE/EFIE/CFIE based on the circular cylinder,' IEEE Trans. Antennas Propag., vol. 53, no. 1, pp. 321-331, Jan. 2005.

[14] M. Carr, E. Topsakal, and J. L. Volakis, "A procedure for modeling material junctions in 3-D surface integral equation approaches," IEEE Trans. Antennas Propag., vol. 52, no. 5, pp. 1374-1379, May 2004.

[15] D. A. Dunavant, "High degree efficient symmetrical Gaussian quadrature rules for the triangle," Int. J. Numer. Meth. Eng., vol. 21, pp. 1129-1148, 1985 
[16] A. W. Glisson and D. R. Wilton, "Simple and efficient numerical methods for problems of electromagnetic radiation and scattering from surfaces," IEEE Trans. Antennas Propag., vol. AP-28, no. 5, pp. 593-603, Sep. 1980.

[17] B. M. Kolundzija and B. D. Popovic, "Entire domain galerkin method for analysis of metallic antennas and scatterers," Proc. Inst. Elect. Eng. Microwaves, Antennas and Propagation, pt. H, vol. 140, no. 1, Feb. 1993.

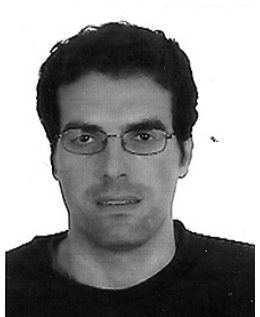

Eduard Ubeda was born in Barcelona, Spain, in 1971. He received the Telecommunication Engineer degree and the Doctor Ingeniero degree from the Universitat Politècnica de Catalunya (UPC), Barcelona, Spain, in 1995 and 2001, respectively.

In 1996, he was with the Joint Research Center, from the European Commission, Ispra, Italy. From 1997 to 2000, he was a Research Assistant at the Electromagnetic and Photonic Engineering group at UPC. From 2001 to 2002, he was a Visiting Scholar in the Electromagnetic Communication Laboratory of the Electrical Engineering Department at the Pennsylvania State University (PSU). Since 2003, he is an Associate Researcher at UPC under the Ramon-y-Cajal programme. He is the author of nine papers in international journals and 20 in international conference proceedings. His main research interests are numerical computation of scattering and radiation using integral equations.

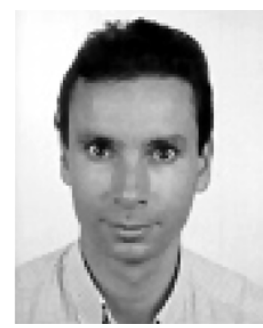

Juan M. Rius (S'89-M'91) received the "Ingeniero de Telecomunicación" degree in 1987 and the "Doctor Ingeniero" degree in 1991, both from the Universitat Politècnica de Catalunya (UPC) at Barcelona.

In 1985, he joined the Electromagnetic and Photonic Engineering group at UPC, in the Department of Signal Theory and Telecommunications (TSC), where he currently holds a position of "Catedrático" (equivalent to Full Professor). From 1985 to 1988, he developed a new inverse scattering algorithm for microwave tomography in cylindrical geometry systems. Since 1989, he has been engaged in the research for new and efficient methods for numerical computation of electromagnetic scattering and radiation. He is the developer of the Graphical Electromagnetic Computation (GRECO) approach for high-frequency RCS computation, the integral equation formulation of the measured equation of invariance (IE-MEI) and the multilevel matrix decomposition algorithm (MLMDA) in 3-D. Current interests are the numerical simulation of electrically large antennas: multiband fractal antennas, large microstrip arrays and reflector antennas. He has held positions of Visiting Professor at EPFL (Lausanne) from May 1, 1996 to October 31, 1996 and April 1, 2001 to June 30, 2001; Visiting Fellow at City University of Hong Kong from January 3, 1997 to February 4, 1997; CLUSTER Chair at EPFL (Lausanne) from December 1, 1997 to January 31, 1998. He has more than 35 publications in refereed international journal papers (16 in IEEE TRANSACTIONS) and more than 100 in international conference proceedings. 